\title{
On order-Lipschitz mappings in Banach spaces without normalities of involving cones
}

\author{
Zhilong Li ${ }^{\mathrm{a}, \mathrm{b}}$, Shujun Jiang ${ }^{\mathrm{c}, *}$, Rade Lazovićd \\ a School of Statistics, Jiangxi University of Finance and Economics, Nanchang, 330013, China. \\ ${ }^{b}$ Research Center of Applied Statistics, Jiangxi University of Finance and Economics, Nanchang, 330013, China. \\ ${ }^{c}$ Department of Mathematics, Jiangxi University of Finance and Economics, Nanchang, 330013, China. \\ ${ }^{d}$ Faculty of Organizational Sciences, University of Belgrade, Jove llica 154, Belgrade, Serbia. \\ Communicated by R. Saadati
}

\begin{abstract}
We prove a new fixed point theorem of order-Lipschitz mappings in Banach spaces without assumption of normalities of the involving cones, which presents a positive answer to a problem raised in [S. Jiang, Z. Li, Fixed Point Theory Appl., 2016 (2016), 10 pages] and improves the corresponding results of Krasnoselskii and Zabreiko's and Zhang and Sun's since the normality of the involving cone is removed. (C)2017 All rights reserved.
\end{abstract}

Keywords: Fixed point theorem, order-Lipschitz mapping, Picard-completeness, non-normal cone. 2010 MSC: 06A07, 47H10.

\section{Introduction and preliminaries}

Let $\mathrm{P}$ be a cone of a Banach space $(E,\|\cdot\|), D \subset E$ and $\preceq$ the partial order in $E$ deduced by $P$. Recall that a mapping $T: D \rightarrow E$ is an order-Lipschitz mapping, if there exist two linear bounded mappings $\mathrm{A}, \mathrm{B}: \mathrm{P} \rightarrow \mathrm{P}$ such that

$$
-B(x-y) \preceq T x-T y \preceq A(x-y), \quad \forall x, y \in D, \quad y \preceq x .
$$

In particular, when $A=B$, Krasnoselskii and Zabreiko [4] proved the following fixed point theorem of order-Lipschitz mappings by using the Banach contraction principle.

Theorem 1.1 ([4]). Let $\mathrm{P}$ be a normal solid cone of a Banach space $(\mathrm{E},\|\cdot\|)$ and $\mathrm{T}: \mathrm{E} \rightarrow \mathrm{E}$ an order-Lipschitz mapping such that (1.1) is satisfied with linear bounded mappings $\mathrm{A}$ and $\mathrm{B}$. If $\mathrm{A}=\mathrm{B}$ and $\|\mathrm{A}\|<1$, then $\mathrm{T}$ has a unique fixed point $x^{*} \in \mathrm{E}$, and $\mathrm{x}_{\mathrm{n}} \stackrel{w}{\rightarrow} x^{*}$ for each $\mathrm{x}_{0} \in \mathrm{E}$, where $\left\{\mathrm{x}_{\mathrm{n}}\right\}=\mathrm{O}\left(\mathrm{T}, \mathrm{x}_{0}\right)$ and $\mathrm{O}\left(\mathrm{T}, \mathrm{x}_{0}\right)$ denotes the Picard iterative sequence of $\mathrm{T}$ at $\mathrm{x}_{0}$, i.e., $\mathrm{x}_{\mathrm{n}}=\mathrm{T}^{\mathrm{n}} \mathrm{x}_{0}$ for each $\mathrm{n}$.

\footnotetext{
*Corresponding author

Email addresses: 1z1771218@sina.com (Zhilong Li), jiangshujuns@sina.com (Shujun Jiang), lazovic@fon.bg.ac.rs (Rade Lazović)

doi:10.22436/jnsa.010.01.03
}

Received 2016-06-16 
Afterward, Zhang and Sun [7] showed Theorem 1.1 is still valid in the case that the spectral radius $r(A)<1$, and obtained the following fixed point result.

Theorem 1.2 ([7]). Let $\mathrm{P}$ be a normal solid cone of a Banach space $(\mathrm{E},\|\cdot\|)$ and $\mathrm{T}: \mathrm{E} \rightarrow \mathrm{E}$ an order-Lipschitz mapping such that (1.1) is satisfied with linear bounded mappings $\mathrm{A}$ and $\mathrm{B}$. If $\mathrm{A}=\mathrm{B}$ and $\mathrm{r}(\mathrm{A})<1$, then $\mathrm{T}$ has a unique fixed point $x^{*} \in \mathrm{E}$, and $\mathrm{x}_{\mathrm{n}} \stackrel{w}{\rightarrow} x^{*}$ for each $\mathrm{x}_{0} \in \mathrm{E}$, where $\left\{\mathrm{x}_{\mathrm{n}}\right\}=\mathrm{O}\left(\mathrm{T}, \mathrm{x}_{0}\right)$ and $\mathrm{O}\left(\mathrm{T}, \mathrm{x}_{0}\right)$ denotes the Picard iterative sequence of $\mathrm{T}$ at $\mathrm{x}_{0}$, i.e., $\mathrm{x}_{\mathrm{n}}=\mathrm{T}^{\mathrm{n}} \mathrm{x}_{0}$ for each $\mathrm{n}$.

In particular when $A, B$ are nonnegative real numbers, Sun [6] proved the following fixed point theorem by using the sandwich theorem in the sense of norm-convergence.

Theorem 1.3 ([6]). Let $\mathrm{P}$ be a normal cone of a Banach space $(\mathrm{E},\|\cdot\|), \mathrm{u}_{0}, v_{0} \in \mathrm{E}$ with $\mathrm{u}_{0} \preceq v_{0}$ and $\mathrm{T}:\left[\mathrm{u}_{0}, v_{0}\right] \rightarrow \mathrm{E}$ an order-Lipschitz mapping such that

$$
u_{0} \preceq T u_{0}, \quad T v_{0} \preceq v_{0},
$$

and (1.1) is satisfied with nonnegative real numbers $\mathrm{A}$ and $\mathrm{B}$. If $\mathrm{A} \in[0,1)$ and $\mathrm{B} \in[0,+\infty)$, then $\mathrm{T}$ has a unique fixed point $x^{*} \in\left[u_{0}, v_{0}\right]$, and $x_{n} \stackrel{w}{\rightarrow} x^{*}$ for each $x_{0} \in\left[u_{0}, v_{0}\right]$, where $\left\{x_{n}\right\}=O\left(T, x_{0}\right)$.

Note that the normality of $P$ in Theorems 1.1 and 1.2 is essential for the completeness of $\left(E,\|\cdot\|_{0}\right)$, where $\|\cdot\|_{0}$ is a new norm in $E$ defined by $\|x\|_{0}=\inf _{\mathfrak{u} \in P}\{\|u\|:-u \preceq x \preceq u\}$, which leads to that the Banach contraction principle is applicable there. And the normality of $\mathrm{P}$ in Theorem 1.3 is essential for ensuring that the sandwich theorem holds in the sense of norm-convergence, which makes an important role in its proof. It is well-known that if $\mathrm{P}$ is non-normal then the sandwich theorem does not hold in the sense of norm-convergence, and consequently, the method used in [6] becomes invalid.

In most of the existing works concerned with fixed point theory of order-Lipschitz mappings, the cone is necessarily assumed to be normal. Recently, Jiang and Li [3] considered fixed point theory of order-Lipschitz mappings without assuming the normality of P. By introducing the concept of Picardcompleteness and using the sandwich theorem in the sense of $w$-convergence, they proved the following fixed point theorem of order-Lipschitz mappings in Banach algebras.

Theorem 1.4 ([3]). Let $\mathrm{P}$ be a solid cone of a Banach algebra $(\mathrm{E},\|\cdot\|), \mathrm{u}_{0}, v_{0} \in \mathrm{E}$ with $\mathrm{u}_{0} \preceq v_{0}$, and $\mathrm{T}:\left[\mathrm{u}_{0}, v_{0}\right] \rightarrow \mathrm{E}$ an order-Lipschitz mapping such that (1.1) and (1.2) are satisfied with $\mathrm{A}, \mathrm{B} \in \mathrm{P}$. If $\mathrm{r}(\mathrm{A})<1$ and $\mathrm{B}=\theta$, then $\mathrm{T}$ has a unique fixed point $x^{*} \in\left[u_{0}, v_{0}\right]$, and $x_{n} \stackrel{w}{\rightarrow} x^{*}$ for each $x_{0} \in\left[u_{0}, v_{0}\right]$, where $\left\{x_{n}\right\}=O\left(T, x_{0}\right)$.

In [3] , the authors failed to improve Theorem 1.1 to the case that the cone is non-normal. Instead, they raised a problem whether the normality of $P$ in Theorem 1.1 could be removed. In the paper, we present a positive answer to this problem, and prove that Theorems 1.1 and 1.2 are still valid without assuming the normality of $P$. In addition, we give an suitable example to show the usability of our theorem.

Let $(E,\|\cdot\|)$ be a Banach space. A nonempty closed subset $P$ of $E$ is a cone, if it is such that $a x+$ by $\in P$ for each $x, y \in P$ and each $a, b \geqslant 0$, and $P \cap(-P)=\{\theta\}$, where $\theta$ is the zero element of $E$. Each cone $P$ of a Banach space $E$ determines a partial order $\preceq$ on $E$ by $x \preceq y \Leftrightarrow y-x \in P$ for each $x, y \in X$. For each $\mathfrak{u}_{0}, v_{0} \in E$ with $\mathfrak{u}_{0} \preceq v_{0}$, we set $\left[\mathfrak{u}_{0}, v_{0}\right]=\left\{u \in E: u_{0} \preceq \mathfrak{u} \preceq v_{0}\right\},\left[u_{0},+\infty\right)=\left\{x \in E: u_{0} \preceq x\right\}$ and $\left(-\infty, v_{0}\right]=\left\{x \in E: x \preceq v_{0}\right\}$. A cone $P$ is solid [1] if intP is nonempty, where intP denotes the interior of $P$. For each $x, y \in E$ with $y-x \in \operatorname{intP}$, we write $x \ll y$.

A cone $P$ is normal [1], if there is some positive number $N$ such that $x, y \in E$ and $\theta \preceq x \preceq y$ implies that $\|x\| \leqslant N\|y\|$, and the minimal $N$ is called a normal constant of $P$. Note that an equivalent condition of a normal cone is that $\inf \{\|x+y\|: x, y \in P$ and $\|x\|=\|y\|=1\}>0$, then a cone $P$ is non-normal, if and only if there exist $\left\{u_{n}\right\},\left\{v_{n}\right\} \subset P$ such that $u_{n}+v_{n} \stackrel{\|\cdot\|}{\rightarrow} \theta \nRightarrow u_{n} \stackrel{\|\cdot\|}{\rightarrow} \theta$. This yields that the sandwich theorem does not hold in the sense of norm-convergence.

Definition 1.5 ([3]). Let $P$ be a solid cone of a Banach space $(E,\|\cdot\|),\left\{x_{n}\right\} \subset E$ and $D \subset E$.

(i) The sequence $\left\{x_{n}\right\}$ is $w$-convergent, if for each $\epsilon \in \operatorname{intP}$, there exist some positive integer $n_{0}$ and $x \in E$ such that $x-\epsilon \ll x_{n} \ll x+\epsilon$ for each $n \geqslant n_{0}$ (denote $x_{n} \stackrel{w}{\rightarrow} x$ and $x$ is called a $w$-limit of $\left.\left\{x_{n}\right\}\right)$; 
(ii) the sequence $\left\{x_{n}\right\}$ is $w$-Cauchy, if for each $\epsilon \in \operatorname{int} P$, there exists some positive integer $n_{0}$ such that $-\epsilon \ll x_{n}-x_{m} \ll \epsilon$ for each $m, n \geqslant n_{0}$, i.e., $x_{n}-x_{m} \stackrel{w}{\rightarrow} \theta(m, n \rightarrow \infty) ;$

(iii) the subset $D$ is $w$-closed, if for each $\left\{x_{n}\right\} \subset D, x_{n} \stackrel{w}{\rightarrow} x$ implies $x \in D$.

The following lemmas are very important for our further discussions.

Lemma 1.6 ([3]). Let $\mathrm{P}$ be a solid cone of a Banach space $(\mathrm{E},\|\cdot\|)$ and $\left\{x_{n}\right\}$ a w-convergent sequence of $\mathrm{E}$. Then $\left\{x_{n}\right\}$ has a unique $w$-limit.

Lemma $1.7([5,2])$. Let $\mathrm{P}$ be a solid cone of a Banach space $(\mathrm{E},\|\cdot\|)$ and $\left\{x_{n}\right\},\left\{y_{n}\right\},\left\{z_{n}\right\} \subset \mathrm{E}$ with $x_{n} \preceq y_{n} \preceq z_{n}$ for each $\mathrm{n}$. If $\mathrm{x}_{\mathrm{n}} \stackrel{w}{\rightarrow} z$ and $z_{\mathrm{n}} \stackrel{w}{\rightarrow} z$, then $\mathrm{y}_{\mathrm{n}} \stackrel{w}{\rightarrow} z$.

Lemma $1.8([5,2])$. Let $\mathrm{P}$ be a solid cone of a Banach space $(\mathrm{E},\|\cdot\|)$ and $\mathrm{x}_{\mathrm{n}} \subset \mathrm{E}$. Then $\mathrm{x}_{\mathrm{n}} \stackrel{\|\cdot\|}{\rightarrow} \mathrm{x}$ implies $\mathrm{x}_{\mathrm{n}} \stackrel{w}{\rightarrow} \mathrm{x}$. Moreover, if $\mathrm{P}$ is normal then $\mathrm{x}_{\mathrm{n}} \stackrel{w}{\rightarrow} \mathrm{x} \Leftrightarrow \mathrm{x}_{\mathrm{n}} \stackrel{\|\cdot\|}{\rightarrow} \mathrm{x}$.

Lemma 1.9 ([1]). Let $\mathrm{P}$ be a solid cone of a Banach space $(\mathrm{E},\|\cdot\|))$. Then there is $\tau>0$ such that for each $\mathrm{x} \in \mathrm{E}$, there exist $y, z \in \mathrm{P}$ with $\|y\| \leqslant \tau\|x\|$ and $\|z\| \leqslant \tau\|x\|$ such that $x=y-z$.

Definition $1.10([3])$. Let $P$ be a solid cone of a Banach space $(E,\|\cdot\|), x_{0} \in E$ and $T: E \rightarrow E$. If the Picard iterative sequence $\mathrm{O}\left(\mathrm{T}, \mathrm{x}_{0}\right)$ is $w$-convergent provided that it $w$-Cauchy, then $\mathrm{T}$ is said to be Picard-complete at $x_{0}$. If $T$ is Picard-complete at each $x \in E$, then it is said to be Picard-complete on $E$.

Remark 1.11.

(i) If $\mathrm{O}\left(\mathrm{T}, \mathrm{x}_{0}\right)$ is $w$-convergent, then $\mathrm{T}$ is certainly Picard-complete at $x_{0}$.

(ii) If $\mathrm{P}$ is a normal cone then each mapping $\mathrm{T}: \mathrm{E} \rightarrow \mathrm{E}$ is Picard-complete on $\mathrm{E}$ by Lemma 1.8.

\section{Main results}

Theorem 2.1. Let $\mathrm{P}$ be a solid cone of a Banach space $(\mathrm{E},\|\cdot\|)$ and $\mathrm{T}: \mathrm{E} \rightarrow \mathrm{E}$ an order-Lipschitz mapping such that (1.1) is satisfied with linear bounded mappings $A$ and $B$. If $A=B, r(A)<1$ and

$$
\mathrm{E}_{\mathrm{T}-\mathrm{C}}=\{\mathrm{x} \in \mathrm{E}: \mathrm{T} \text { is Picard-complete at } \mathrm{x}\} \neq \varnothing,
$$

then $\mathrm{T}$ has a unique fixed point $\mathrm{x}^{*} \in \mathrm{E}$. Moreover, for each $\mathrm{x}_{0} \in \mathrm{E}_{\mathrm{T}-\mathrm{C}}$, we have $\mathrm{x}_{\mathrm{n}} \stackrel{w}{\rightarrow} x^{*}$, where $\left\{\mathrm{x}_{\mathrm{n}}\right\}=\mathrm{O}\left(\mathrm{T}, \mathrm{x}_{0}\right)$. Proof.

Step 1. We show that for each $x, y \in X$, there exists $u \in P$ such that

$$
-u \preceq x-y \preceq u,
$$

and

$$
-A^{n} u \preceq T^{n} x-T^{n} y \preceq A^{n} u, \quad \forall n .
$$

It follows from the solidness of $P$ and Lemma 1.9 that there is a $\tau>0$ such that for each $x \in E$, there exist $y, z \in P$ with $\|y\| \leqslant \tau\|x\|$ and $\|z\| \leqslant \tau\|x\|$ such that $x=y-z$, and so we have

$$
-(y+z) \preceq x \preceq y+z .
$$

This shows that for each $x \in E$, there exists $u \in P$ such that

$$
-u \preceq x \preceq u,
$$


and so for each $x, y \in E$, there exists $u \in P$ such that (2.1) is satisfied. For each $x, y \in E$, by (2.1) we get

$$
\frac{x+y-u}{2} \preceq x, \quad \frac{x+y-u}{2} \preceq y .
$$

Thus by (1.1), we have

$$
-A\left(\frac{x-y+u}{2}\right) \preceq T x-T\left(\frac{x+y-u}{2}\right) \preceq A\left(\frac{x-y+u}{2}\right),
$$

and

$$
-A\left(\frac{y-x+u}{2}\right) \preceq T y-T\left(\frac{x+y-u}{2}\right) \preceq A\left(\frac{y-x+u}{2}\right),
$$

which can be rewritten as

$$
-A\left(\frac{y-x+u}{2}\right) \preceq T\left(\frac{x+y-u}{2}\right)-T y \preceq A\left(\frac{y-x+u}{2}\right) .
$$

By adding (2.3) and (2.4), we get

$$
-A u \preceq T x-T y \preceq A u,
$$

i.e., (2.2) holds for $n=1$. Suppose that (2.2) holds for $n$, then

$$
\frac{T^{n} x+T^{n} y-A^{n} u}{2} \preceq T^{n} x, \quad \frac{T^{n} x+T^{n} y-A^{n} u}{2} \preceq T^{n} y .
$$

Moreover by (1.1), we have

$$
-A\left(\frac{T^{n} x-T^{n} y+A^{n} u}{2}\right) \preceq T^{n+1} x-T\left(\frac{T^{n} x+T^{n} y-A^{n} u}{2}\right) \preceq A\left(\frac{T^{n} x-T^{n} y+A^{n} u}{2}\right),
$$

and

$$
-A\left(\frac{T^{n} y-T^{n} x+A^{n} u}{2}\right) \preceq T^{n+1} y-T\left(\frac{T^{n} x+T^{n} y-A^{n} u}{2}\right) \preceq A\left(\frac{T^{n} y-T^{n} x+A^{n} u}{2}\right),
$$

which can be rewritten as

$$
-A\left(\frac{T^{n} y-T^{n} x+A^{n} u}{2}\right) \preceq T\left(\frac{T^{n} x+T^{n} y-A^{n} u}{2}\right)-T^{n+1} y \preceq A\left(\frac{T^{n} y-T^{n} x+A^{n} u}{2}\right) .
$$

By adding (2.5) and (2.6), we get $-A^{n+1} \mathfrak{u} \preceq T^{n+1} x-T^{n+1} y \preceq A^{n+1} u$ for each $x, y \in E$, i.e., (2.2) holds for $n+1$. Thus (2.2) holds true by induction.

Step 2. We show that there exists a positive integer $n_{0}$ such that $T^{n_{0}}$ has a unique fixed point in $E$.

By $r(A)<1, I-A$ is invertible, denote the inverse of $I-A$ by $(I-A)^{-1}$. Moreover, it follows from Neumann's formula that

$$
(I-A)^{-1}=\sum_{n=0}^{\infty} A^{n}=I+A+A^{2}+\cdots+A^{n}+\cdots,
$$

which implies that $(I-A)^{-1}: P \rightarrow P$ is a linear bounded mapping. It follows from $r(A)<1$ and Gelfand's formula that there exists a positive integer $n_{1}$ and $\beta \in(r(A), 1)$ such that

$$
\left\|A^{n}\right\| \leqslant \beta^{n}, \quad \forall n \geqslant n_{1} .
$$

Thus for each $u \in P$, we get

$$
\left\|A^{n} u\right\| \leqslant\left\|A^{n}\right\|\left\|u \mid \leqslant \beta^{n}\right\| u \|, \quad \forall n \geqslant n_{1},
$$

which implies $A^{n} u \stackrel{\|\cdot\|}{\rightarrow} \theta$ for each $u \in P$, and hence by Lemma 1.8,

$$
A^{n} u \stackrel{w}{\rightarrow} \theta, \quad \forall u \in P .
$$


Since $(I-A)^{-1}: P \rightarrow P$ is a linear bounded mapping, in analogy to (2.9), by (2.8) we obtain

$$
A^{n}(I-A)^{-1} u \stackrel{w}{\rightarrow} \theta, \quad \forall u \in P .
$$

Let $x_{0} \in E_{T-C}$ and set $\left\{x_{n}\right\}=O\left(T, x_{0}\right)$, then by Step 1, there exists $u_{x_{0}, x_{1}} \in P$ such that

$$
-u_{x_{0}, x_{1}} \preceq x_{0}-x_{1} \preceq u_{x_{0}, x_{1}}
$$

and

$$
-A^{n} u_{x_{0}, x_{1}} \preceq x_{n+1}-x_{n}=T^{n} x_{1}-T^{n} x_{0} \preceq A^{n} u_{x_{0}, x_{1}}, \quad \forall n .
$$

Thus by (2.7), for each $m>n$ we have

$$
\begin{aligned}
-A^{n}(I-A)^{-1} u_{x_{0}, x_{1}} & \preceq-\sum_{i=n}^{m-1} A^{i} u_{x_{0}, x_{1}} \preceq x_{m}-x_{n}=\sum_{i=n}^{m-1}\left(x_{i+1}-x_{i}\right) \preceq \sum_{i=n}^{m-1} A^{i} u_{x_{0}, x_{1}} \\
& \preceq A^{n}(I-A)^{-1} u_{x_{0}, x_{1}},
\end{aligned}
$$

which together with (2.10) and Lemma 1.7 implies that

$$
x_{m}-x_{n} \stackrel{w}{\rightarrow} \theta(m>n \rightarrow \infty),
$$

i.e., $\left\{x_{n}\right\}$ is $w$-Cauchy. Note that $T$ is Picard-complete at $x_{0}$, then there exists some $x^{*} \in E$ such that

$$
x_{\mathrm{n}} \stackrel{w}{\rightarrow} x^{*}(\mathrm{n} \rightarrow \infty)
$$

By Step 1 , there exists $u_{x_{0}, x^{*}} \in P$ such that $-u_{x_{0}, x^{*}} \preceq x_{0}-x^{*} \preceq u_{x_{0}, x^{*}}$ and

$$
-A^{n} u_{x_{0}, x^{*}} \preceq x_{n}-T^{n} x^{*}=T^{n} x_{0}-T^{n} x^{*} \preceq A^{n} u_{x_{0}, x^{*}}
$$

which together with (2.9) and Lemma 1.7 implies that

$$
x_{\mathrm{n}}-\mathrm{T}^{\mathrm{n}} \chi^{*} \stackrel{w}{\rightarrow} \theta(\mathrm{n} \rightarrow \infty) .
$$

For each $\epsilon \in \operatorname{intP}$, it follows from (2.11) and (2.13) that there exists a positive integer $n_{0}$ such that

$$
-\frac{\epsilon}{2} \ll x_{m}-x_{n} \ll \frac{\epsilon}{2}, \quad \forall m>n \geqslant n_{0},
$$

and

$$
-\frac{\epsilon}{2} \ll x_{n}-\mathrm{T}^{\mathrm{n}} \chi^{*} \ll \frac{\epsilon}{2}, \quad \forall \mathrm{n} \geqslant \mathrm{n}_{0}
$$

Thus by (2.14) and (2.15) we get

$$
-\epsilon \ll x_{m}-T^{n_{0}} \chi^{*}=x_{m}-x_{n_{0}}+x_{n_{0}}-T^{n_{0}} x^{*} \ll \epsilon, \quad \forall m>n_{0},
$$

and hence

$$
x_{\mathrm{m}} \stackrel{w}{\rightarrow} \mathrm{T}^{\mathrm{n}_{0}} \chi^{*}(\mathrm{~m} \rightarrow \infty) .
$$

Moreover by Lemma 1.6, we get $x^{*}=T^{n_{0}} x^{*}$, since $\left\{x_{n}\right\}$ has a unique $w$-limit. Suppose that $z$ is a fixed point of $T^{n_{0}}$, then by Step 1 , there exists $\mathfrak{u}_{z, x^{*}}$ such that $-u_{z, x^{*}} \preceq z-x^{*} \preceq u_{z, x^{*}}$ and

$$
-A^{n n_{0}} u_{z, x^{*}} \preceq z-x^{*}=T^{n n_{0}} z-T^{n n_{0}} x^{*} \preceq A^{n n_{0}} u_{z, x^{*}}, \quad \forall n,
$$

which together with (2.9) and Lemma 1.7 implies that $z=x^{*}$. Hence $x^{*}$ is the unique fixed point of $\mathrm{T}^{\mathrm{n}_{0}}$. 
Step 3. We show that $x^{*}$ is the unique fixed point of $T$.

Note that $T^{n_{0}}\left(T x^{*}\right)=T^{n_{0}+1} \chi^{*}=T\left(T^{n_{0}} x\right)=T x^{*}$, then $T x^{*}$ is a fixed point of $T^{n_{0}}$, and hence $x^{*}=T x^{*}$ by the uniqueness of fixed point of $T^{n_{0}}$. This shows $x^{*}$ is a fixed point of $T$. Suppose that $z \in E$ is a fixed point of $\mathrm{T}$, then $z$ is a fixed point of $\mathrm{T}^{\mathrm{n}_{0}}$, and hence $z=x^{*}$ by the unique existence of fixed point of $\mathrm{T}^{\mathrm{n}_{0}}$. Hence $x^{*}$ is the unique fixed point of $T$.

Example 2.2. Let $E=C_{\mathbb{R}}^{1}[0,1]$ be endowed with the norm $\|x\|=\|x\|_{\infty}+\left\|x^{\prime}\right\|_{\infty}$ and

$$
\mathrm{P}=\{x \in \mathrm{E}: x(\mathrm{t}) \geqslant 0, \forall \mathrm{t} \in[0,1]\},
$$

where $\|x\|_{\infty}=\max _{t \in[0,1]} x(t)$ for each $x \in C_{\mathbb{R}}[0,1]$. Then $(E,\|\cdot\|)$ is a Banach space and $P$ is a non-normal solid cone [1]. Let $x_{0}(t) \equiv \frac{1}{2}, D=\left\{x \in E:\|x\| \leqslant \frac{1}{2}\right\}$ and $(T x)(t)=\int_{0}^{t} x^{2}(s) d s$ for each $x \in E$ and each $\mathrm{t} \in[0,1]$. Clearly, $x_{0} \in \mathrm{D}$ and $\mathrm{T}(\mathrm{D}) \subset \mathrm{D}$ since $\|\mathrm{T} x\|=\|\mathrm{T} x\|_{\infty}+\left\|(T x)^{\prime}\right\|_{\infty} \leqslant \frac{1}{2}$ for each $x \in \mathrm{D}$.

Set $\left\{x_{n}\right\}=O\left(T, x_{0}\right)$. By induction we get

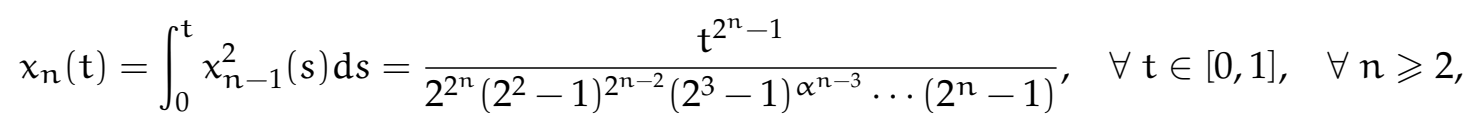

and so

$$
\theta \preceq x_{n} \preceq \frac{1}{2^{2^{n}}\left(2^{2}-1\right)^{2^{n-2}}\left(2^{3}-1\right)^{\alpha^{n-3} \cdots\left(2^{n}-1\right)}}, \quad \forall n \geqslant 2 ，
$$

which together with Lemma 1.7 implies that $x_{n} \stackrel{w}{\rightarrow} \theta$. Moreover by (i) of Remark 1.11, we know that $\mathrm{T}$ is Picard-complete at $x_{0}$.

For each $x, y \in D$ with $y \preceq x$ and each $t \in[0,1]$, we have

$$
-\int_{0}^{t}(x(s)-y(s)) d s \leqslant(T x)(t)-(T y)(t)=\int_{0}^{t}(x(s)-y(s))(x(s)+y(s)) d s \leqslant \int_{0}^{t}(x(s)-y(s)) d s,
$$

and so

$$
-A(x-y) \preceq T x-T y \preceq A(x-y), \quad \forall x, y \in D, \quad y \preceq x,
$$

where $(A x)(t)=\int_{0}^{t} x(s) d s$ for each $x \in E$ and each $t \in[0,1]$. This shows that $T: D \rightarrow D$ is an orderLipschitz mapping.

For each $x \in E$ and $t \in[0,1]$, by induction we get $\left(A^{n} x\right)(t) \leqslant \frac{\|x\|_{\infty} t^{n}}{n !} \leqslant \frac{\|x\|}{n !}$, and so $\left\|A^{n} x\right\|_{\infty} \leqslant \frac{\|x\|}{n !}$. On the other hand, we have $\left\|\left(A^{n} x\right)^{\prime}\right\|_{\infty}=\left\|A^{n-1} x\right\|_{\infty} \leqslant \frac{\|x\|}{(n-1) !}$ since $\left(A^{n} x\right)^{\prime}(t)=\left(A^{n-1} x\right)(t)$. Thus $\left\|A^{n} x\right\|=\left\|A^{n} x\right\|_{\infty}+\left\|\left(A^{n} x\right)^{\prime}\right\|_{\infty} \leqslant \frac{\|x\|}{n !}+\frac{\|x\|}{(n-1) !}$ and $\left\|A^{n}\right\| \leqslant \frac{1}{n !}+\frac{1}{(n-1) !}$. By Gelfand's formula, we obtain $r(A)=\lim _{n \rightarrow \infty} \sqrt[n]{\frac{1}{n !}+\frac{1}{(n-1) !}} \leqslant \lim _{n \rightarrow \infty} \frac{1}{\sqrt[n]{n !}}+\lim _{n \rightarrow \infty} \frac{1}{\sqrt[n]{(n-1) !}}=0$. Therefore $T: D \rightarrow D$ has a unique fixed point in $\mathrm{D}$ by Theorem 2.1 (in fact, $\theta$ is the unique fixed point of $\mathrm{T}$ ).

However, Theorems 1.1, 1.2, 1.3 and 1.4 are not applicable here since $P$ is non-normal and there do not exist $A, B \in P$ or nonnegative real numbers $A, B$ such that (1.1) is satisfied.

Remark 2.3. Theorem 2.1 implies that Theorems 1.1 and 1.2 are still valid in the case that $\mathrm{P}$ is non-normal, and hence Theorem 2.1 improves Theorems 1.1 and 1.2. In fact, Theorems 1.1 and 1.2 are immediate consequences of Theorem 2.1 by Remark 1.11 (ii). 2.1.

In particular when $E$ is a Banach algebra and $A, B \in P$, we have the following corollary by Theorem

Corollary 2.4. Let $\mathrm{P}$ be a solid cone of a Banach algebra $(\mathrm{E},\|\cdot\|)$ and $\mathrm{T}: \mathrm{E} \rightarrow \mathrm{E}$ an order-Lipschitz mapping such that (1.1) is satisfied with $\mathrm{A}, \mathrm{B} \in \mathrm{P}$. If $\mathrm{A}=\mathrm{B}, \mathrm{r}(\mathrm{A})<1$ and $\mathrm{E}_{\mathrm{T}-\mathrm{C}}$ is nonempty, where

$$
\mathrm{E}_{\mathrm{T}-\mathrm{C}}=\{\mathrm{x} \in \mathrm{E}: \mathrm{T} \text { is Picard-complete at } \mathrm{x}\},
$$

then $\mathrm{T}$ has a unique fixed point $\mathrm{x}^{*} \in \mathrm{E}$. Moreover, for each $\mathrm{x}_{0} \in \mathrm{E}_{\mathrm{T}-\mathrm{C}}$, we have $\mathrm{x}_{\mathrm{n}} \stackrel{w}{\rightarrow} \mathrm{x}^{*}$, where $\left\{\mathrm{x}_{\mathrm{n}}\right\}=\mathrm{O}\left(\mathrm{T}, \mathrm{x}_{0}\right)$. 
Remark 2.5. It is clear that Theorem 5 in [3] is a particular case of our Corollary 2.4 with normal cones. Note that if (1.1) is satisfied with $A \in P$ and $B=\theta$ then $T:\left[u_{0}, v_{0}\right] \rightarrow E$ is nondecreasing, and hence Corollary 2.4 partially improves Theorem 1.4 since (1.2) and the nondecreasing property of $\mathrm{T}$ are not assumed.

\section{Acknowledgment}

The work was supported by the National Natural Science Foundation of China $(11161022,11561026$, 71462015), the Natural Science Foundation of Jiangxi Province (20142BCB23013,20143ACB21012, 20151BAB201003,20151BAB201023), and the Natural Science Foundation of Jiangxi Provincial Education Department (KJLD14034, GJJ150479).

\section{References}

[1] K. Deimling, Nonlinear functional analysis, Springer-Verlag, Berlin, (1985). 1, 1.9, 2.2

[2] S. Jiang, Z. Li, Extensions of Banach contraction principle to partial cone metric spaces over a non-normal solid cone, Fixed Point Theory Appl., 2013 (2013), 9 pages. 1.7, 1.8

[3] S. Jiang, Z. Li, Fixed point theorems of order-Lipschitz mappings in Banach algebras, Fixed Point Theory Appl., 2016 (2016), 10 pages. 1, 1.4, 1, 1.5, 1.6, 1.10, 2.5

[4] M. A. Krasnosel'skiǔ, P. P. Zabreǐko, Geometrical methods of nonlinear analysis, Springer-Verlag, Berlin, (1984). 1, 1.1

[5] Z. Li, S. Jiang, Common fixed point theorems of contractions in partial cone metric spaces over nonnormal cones, Abstr. Appl. Anal., 2014 (2014), 8 pages.1.7, 1.8

[6] J.-X. Sun, Iterative solutions of nonlinear operator, (Chinese) J. Engin. Math., 6 (1989), 12-17. 1, 1.3, 1

[7] X. Y. Zhang, J. X. Sun, Existence and uniqueness of solutions for a class of nonlinear operator equations and its applications, (Chinese) Acta Math. Scientia, 25 (2005), 846-851. 1, 1.2 